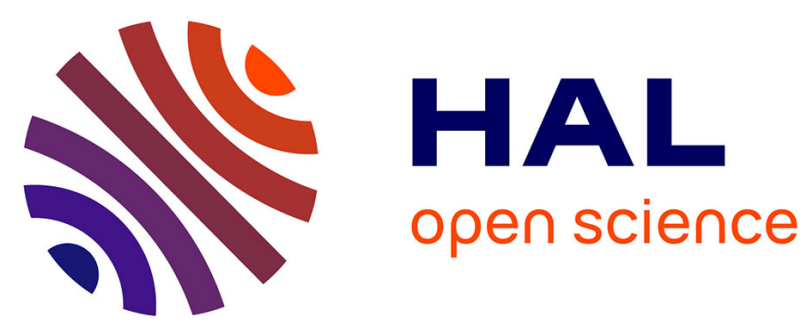

\title{
Contrôle de la natalité et changement social des familles paysannes de fronts pionniers d'Amazonie orientale brésilienne
}

Xavier Arnauld de Sartre, Hélène Guetat-Bernard, Gutemberg Guerra

\section{- To cite this version:}

Xavier Arnauld de Sartre, Hélène Guetat-Bernard, Gutemberg Guerra. Contrôle de la natalité et changement social des familles paysannes de fronts pionniers d'Amazonie orientale brésilienne. Autrepart - Revue de sciences sociales au Sud, 2006, 39, pp.21-38. halshs-00453864

\section{HAL Id: halshs-00453864 \\ https://shs.hal.science/halshs-00453864}

Submitted on 5 Jan 2022

HAL is a multi-disciplinary open access archive for the deposit and dissemination of scientific research documents, whether they are published or not. The documents may come from teaching and research institutions in France or abroad, or from public or private research centers.
L'archive ouverte pluridisciplinaire HAL, est destinée au dépôt et à la diffusion de documents scientifiques de niveau recherche, publiés ou non, émanant des établissements d'enseignement et de recherche français ou étrangers, des laboratoires publics ou privés. 


\title{
CONTRÔLE DE LA NATALITÉ ET CHANGEMENT SOCIAL DES FAMILLES PAYSANNES DE FRONTS PIONNIERS D'AMAZONIE ORIENTALE BRÉSILIENNE
}

\author{
Xavier Arnauld de Sartre et al. \\ Presses de Sciences Po | Autrepart
}

$2006 / 3-n^{\circ} 39$

pages 21 à 38

ISSN 1278-3986

Article disponible en ligne à l'adresse:

http:/www.cairn.info/revue-autrepart-2006-3-page-21.htm

Pour citer cet article :

de Sartre Xavier Arnauld et al., "Contrôle de la natalité et changement social des familles paysannes de fronts pionniers d'Amazonie orientale brésilienne »,

Autrepart, 2006/3 n³9, p. 21-38. DOI : 10.3917/autr.039.0021

Distribution électronique Cairn.info pour Presses de Sciences Po.

(C) Presses de Sciences Po. Tous droits réservés pour tous pays.

La reproduction ou représentation de cet article, notamment par photocopie, n'est autorisée que dans les limites des conditions générales d'utilisation du site ou, le cas échéant, des conditions générales de la licence souscrite par votre établissement. Toute autre reproduction ou représentation, en tout ou partie, sous quelque forme et de quelque manière que ce soit, est interdite sauf accord préalable et écrit de l'éditeur, en dehors des cas prévus par la législation en vigueur en France. II est précisé que son stockage dans une base de données est également interdit. 


\title{
Contrôle de la natalité et changement social des familles paysannes de fronts pionniers d'Amazonie orientale brésilienne ${ }^{1}$
}

\author{
Xavier Arnauld de Sartre*, Hélène Guétat-Bernard**, \\ Gutemberg Armando Diniz Guerra***
}

\section{Introduction}

Durant les années 1970, le souci de maîtrise de l'espace par l'État militaire explique la rapide expansion des fronts de colonisation de l'Amazonie orientale brésilienne. Aux côtés de facteurs agro-économiques, les logiques sociales de reproduction des familles paysannes jouent aussi un rôle non négligeable dans la dynamique de transformation du couvert forestier [Léna, 1999]. Nous questionnons précisément les conséquences de la forte chute de la fécondité des familles paysannes (du Sud ou du Nordeste) sur la dynamique spatiale. Nous interrogeons surtout certains moments cruciaux du cycle de leur vie, comme les temps de fission des groupes domestiques ou de renouvellement des générations. Parce que ces temps sont révélateurs des relations entre les sexes et entre les générations [Segalen, 1980], les relations de genre s'invitent dans les débats : en permettant de repérer des conflits de représentation des modes de reproduction de ces familles, l'analyse des rapports hommes-femmes permet de mettre en évidence plus facilement les changements.

1. Ce travail s'intègre dans trois programmes de recherche :

Zone Atelier de recherche sur l'environnement, CNRS, Programme Environnement, vie et sociétés, «Environnement et développement en front pionnier amazonien : étude de sites et d'expériences pour une coévolution de l'agriculture familiale avec son environnement »;

Programme PREMER, MAE, « Nouvelles compétences pour l'innovation localisée et le développement durable des territoires ruraux. Projet de coopération France / Argentine / Brésil au niveau Master »;

Programme « EBIMA, Enquêtes biographiques en Amazonie », de l'Action concertée incitative Sociétés et cultures dans le développement durable.

* Géographe, Chargé de recherches au CNRS, UMR Société Environnement Territoire (UMR 5603 CNRS / Univ. Pau), IRSAM, Université de Pau, 64000 Pau, xavier.arnauld@univ-pau.fr

** Géographe, enseignant chercheur, UMR Dynamiques Rurales, MDR, Université de Toulouse 2, 5 allées Antonio Machado, 31058 Toulouse Cedex 9, phguetat@aol.com

*** Sociologue, Enseignant chercheur, Département d'Etudes Intégrées sur l'Agriculture Familiale, Université Fédérale du Pará (Brésil), Campus de Guama, CEP 66075-110 - Caixa postal 479, Belém / Pará / Brésil, gute@amazon.com.br 
Ces changements ne peuvent être caractérisés que si l'on connaît les modes de fonctionnement qui leur préexistaient. Si la catégorie «d'agriculture familiale » s'est imposée au Brésil pour qualifier ces familles, cette catégorie, trop vague, a l'inconvénient de réduire la grande diversité sociologique qu'elle recoupe [Brumer et al., 2001]. Nous préférons dès lors qualifier plus précisément les logiques des agriculteurs que nous avons rencontrés, en recourrant à une typologie qui oppose d'un côté des agriculteurs citadins, fortement intégrés à la société urbaine et dont les comportements sociaux sont les mêmes que ceux des citadins, aux paysans, beaucoup plus autonomes à l'égard de la société englobante et dont les logiques sociales peuvent se comprendre autour du triptyque famille - exploitation - terre [Mendras, 2000 ; Arnauld de Sartre, 2006]. Les paysans ont jusqu'alors échappé à la transition démographique : on peut en trouver les raisons autant dans l'autonomie vis-à-vis de la société nationale que dans le fonctionnement interne de ces familles, pour lesquelles la main-d'œuvre familiale sur l'exploitation agricole est essentielle et qui disposent, par le biais de la migration vers de nouveaux fronts pionniers, de facilités pour obtenir de la terre - et donc doter leurs enfants.

Or la réduction significative de la fécondité, tout en favorisant une baisse de la pression foncière exercée directement par les agriculteurs, révèle des changements de mentalité dans les fronts pionniers qui obligent à reconsidérer les interdépendances entre les sociétés paysannes des fronts pionniers et la société nationale. Nous présentons dans un premier temps les éléments chiffrés de cette baisse avant d'en proposer une interprétation. Nous abordons ainsi, dans un second temps, les significations des conflits au sein des couples, car cette baisse est aussi révélatrice de tensions sociales. La réduction de la natalité et la maîtrise de la fécondité féminine étant un enjeu de pouvoir majeur [Héritier, 1996], elle est particulièrement révélatrice de changements de la place des femmes dans les couples. Dans un troisième temps, enfin, nous proposons un questionnement sur l'avenir des rapports traditionnels entre l'exploitation agricole, la famille paysanne et la terre qu'elle exploite dans le contexte des fronts de colonisation étudiés.

\section{La réduction de la natalité en Amazonie : interpréter un fait connu et massif}

La région de Marabá, en Amazonie orientale, est emblématique de ces fronts pionniers consacrés à la grande exploitation capitaliste (mines, élevage et production hydroélectrique) dont sont exclues les populations locales, petits agriculteurs en tête [Hall, 1989]. De ce fait, la colonisation est très extensive, privilégiant les moyens de transport modernes et fondant la rentabilité économique sur un dumping environnemental et social. Les différents types d'agriculteurs familiaux ne peuvent de ce fait accéder à la terre que loin des axes routiers, aux limites des grandes exploitations d'élevage. Cet isolement relatif des agriculteurs peut à lui seul expliquer le relatif retard que l'on observe dans leurs comportements démographiques. 


\section{L'entrée dans la transition démographique}

Le Brésil est un pays qui, globalement, se situe en stade intermédiaire de sa transition démographique : le taux moyen de fécondité est de 2,38 enfants par femme, mais avec de fortes inégalités régionales. Ces taux baissent fortement : le taux de fécondité de la population brésilienne est passé de 2,89 à 2,38 enfants par femme entre 1991 et 2000. Avec un certain retard, la transition démographique touche aussi les fronts pionniers : si ceux-ci sont globalement caractérisés par une fécondité supérieure à celle de la moyenne nationale, différents observateurs mettent en évidence le fait qu'elle change depuis le milieu des années 1980 [Hamelin, 1992 ; Léna et Maciel da Silveira, 1993].

Lors d'une enquête menée en Amazonie en 2004, nous avons constaté un tel phénomène. Le graphique 1 représente la fécondité des femmes de deux générations rencontrées en Amazonie orientale [Sebille et al., 2005] ${ }^{2}$.

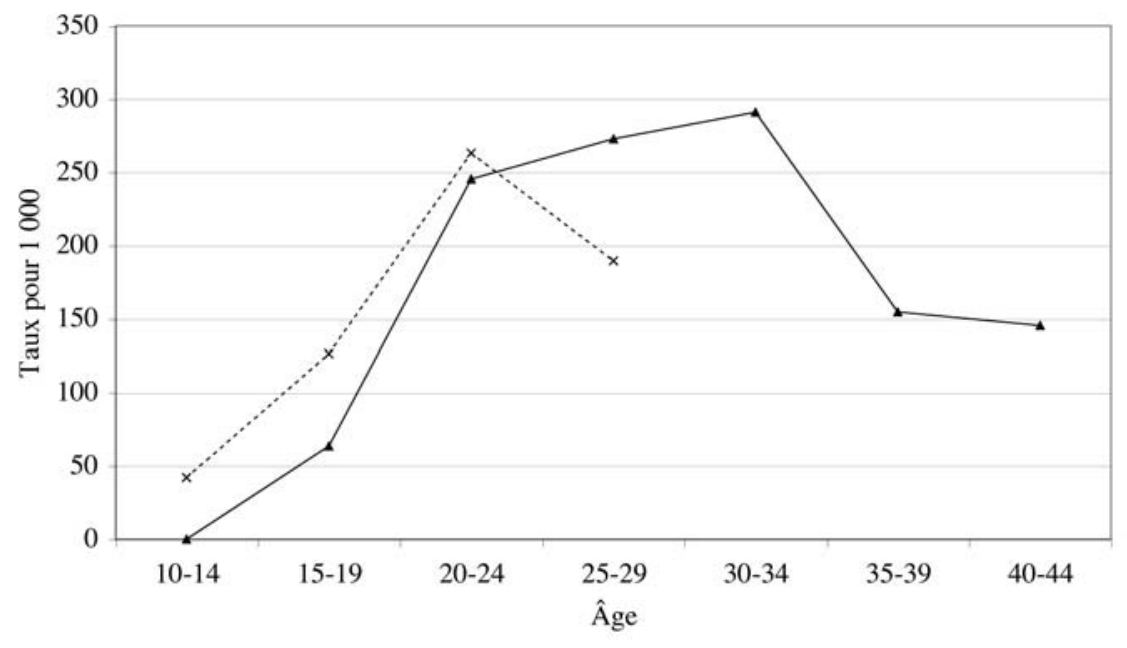

—-Femmes nées avant $1960 \cdots \cdots \cdots$ Femmes nées après 1960

Graphique 1 - Réduction de la fécondité dans les fronts pionniers

Même si les femmes nées après 1960 n'ont pas toutes fini leur vie féconde, on observe un changement de patron démographique. La vie féconde des femmes de la génération la plus jeune commence plus tôt, mais finit beaucoup plus tôt que

2. L'échantillon est composé de 111 individus, qui se répartissent de la manière suivante : 59 individus nés avant 1959, 52 nés après. Pour des raisons de représentativité des effectifs, nous n'avons pas pu faire de cohortes regroupant des classes d'âge plus petites. 
celle de la génération précédente : en effet, alors que ces dernières avaient une fécondité qui atteignait son maximum lorsqu'elles avaient entre 30 et 34 ans, celle des femmes les plus jeunes chute brutalement entre 25 et 29 ans. Ainsi, alors que la forme de la courbe de natalité des femmes les plus âgées est typiquement celle d'une fécondité pré-transitionnelle, celle des femmes les plus jeunes porte la marque de la transition démographique.

\section{La réduction de la natalité : un phénomène non généralisé}

Une étude de la manière dont la transition démographique se passe au niveau familial permet de préciser le phénomène et d'apporter quelques précisions. Sur la base du même échantillon que celui qui a servi à construire le graphique 1 , nous avons construit le tableau 1 . Ce tableau résume par différents indicateurs statistiques le nombre d'enfants par famille et par cohorte, ainsi que la différence entre le nombre d'enfants de notre informateur de celui de ses frères et sœurs (nés de la même mère).

Tab. 1 - Réduction différenciée de la fécondité selon les générations

\begin{tabular}{|l|c|c|c|c|}
\cline { 2 - 5 } \multicolumn{1}{c|}{} & \multicolumn{3}{c|}{ Nombre enfants d'égo } & \multicolumn{2}{c|}{$\begin{array}{c}\text { Différence nombre d'enfants } \\
\text { d'égo - nombre d'enfants } \\
\text { qu'a eu sa mère }\end{array}$} \\
\cline { 2 - 5 } \multicolumn{1}{c|}{} & $\begin{array}{c}\text { Femmes nées } \\
\text { avant } 1960\end{array}$ & $\begin{array}{c}\text { Femmes nées } \\
\text { après 1960 }\end{array}$ & $\begin{array}{c}\text { Femmes nées } \\
\text { avant 1960 }\end{array}$ & $\begin{array}{c}\text { Femmes nées } \\
\text { après 1960 }\end{array}$ \\
\hline Mini & 0 & 0 & -20 & -20 \\
\hline Maxi & 13 & 7 & 12 & 5 \\
\hline Moyenne & 6 & 3 & -2 & -4 \\
\hline Médiane & 6 & 3 & -1 & 4,8 \\
\hline Écart type & 2,8 & 1,8 & 5,3 & -5 \\
\hline
\end{tabular}

Ce tableau permet de voir comment la réduction de la fécondité s'opère sur deux générations. Certes, toutes les femmes nées après 1960 n’ont pas fini leur vie féconde; cependant, le graphique 1 montre que la chute de la fécondité commence, pour cette cohorte, après 25 ans - ce qui laisse supposer que l'écart observé ne sera pas rattrapé. On peut alors considérer que le nombre moyen d'enfants est divisé par deux d'une génération à l'autre, et que ce phénomène est inscrit dans le temps : les femmes nées avant 1960 ont en moyenne deux enfants de moins que leurs parents, mais trois de plus que celles nées après 1960. L'écart type lui aussi baisse d'une génération à l'autre, mais montre qu'aujourd'hui la plus grande partie des familles paysannes ont entre un et cinq enfants. Cela renvoie à des disparités importantes selon les familles et à l'intérieur des couples - différences qui, expliquées, permettent de comprendre la manière dont la natalité est réduite. 
L'entretien plurigénérationnel d'époux séparés :

le domaine de l'alcôve relève du caché et de l'intime

Les chiffres présentés ci-dessus sont-ils plus le fruit d'un consensus obtenu au sein du couple devant le chercheur que la révélation d'une planification rigide? Pour répondre à cette question, interroger les rapports entre les époux est essentiel pour expliquer le nombre d'enfants par famille. Le schéma suivant propose une matrice théorique des rapports entre les époux quant à la réduction du nombre d'enfants.

\section{Rapport à la réduction du nombre d'enfants}

\begin{tabular}{cccc} 
& & \multicolumn{2}{c}{ Homme } \\
& & Favorable & $\begin{array}{c}\text { Pas } \\
\text { favorable }\end{array}$ \\
& & & 2 \\
Femme & Favorable & 1 & \\
\cline { 3 - 4 } & Pas & 3 & 4 \\
& favorable & &
\end{tabular}

1. Réduction du nombre d'enfants

2. Conflit très fort

3. Jamais observé

4. Maintien d'un nombre élevé d'enfants, mais avec quand même réduction

Lorsque maris et femmes sont favorables à une réduction du nombre d'enfants, celle-ci se produit inévitablement - la femme réalisant une ligature des trompes une fois le nombre maximum d'enfants souhaités atteint. Mais cette situation s'est révélée, a priori (nous serons amené au cours de cet article à déconstruire cette affirmation), peu révélatrice des causes menant à la réduction du nombre d'enfants. De même les discours du type : «avoir moins d'enfants, c'est nécessaire pour mieux pouvoir s'en occuper », ou, inversement, « on souhaite de nombreux enfants parce qu'on les aime » ne peuvent apparaître comme des explications suffisantes. En effet, ils énoncent des vérités générales difficiles à contredire autant qu'à vérifier. Les situations de conflits, pas généralisées mais suffisamment nombreuses pour pouvoir être étudiées en tant que telles, sont apparues au contraire particulièrement révélatrices des manières différenciées pour l'époux et l'épouse de se représenter leur place dans le couple et la société paysanne.

Le protocole de recherche mis en place a permis de rencontrer séparément le mari et l'épouse pour éviter au maximum (de part et d'autre) les propos sous contrainte. Cette proposition méthodologique a permis de confronter les regards sur un même questionnement, et de comparer les connaissances de l'un et de l'autre sur 
les sujets concernant l'exploitation et la famille. On a ainsi pu voir émerger des questionnements plus préoccupants pour l'un que pour l'autre. Ces entretiens semi directifs, qui portent au total sur une soixantaine de familles, ont tous été enregistrés, transcrits et analysés. Ils se présentaient sous la forme de récits de vie (entretiens biographiques) où la question de la fécondité était abordée comme un thème parmi d'autres. La réduction de la fécondité est alors replacée dans un contexte plus global. Cela est particulièrement le cas lorsqu'il y a des conflits. De même, le choix méthodologique de rencontrer, dans une même famille (lorsque cela était possible) deux générations de paysans mariés, nous a permis de comprendre qu'en termes de maîtrise de la fécondité, les revendications féminines actuelles mais aussi les positionnements masculins favorables à une réduction des naissances, peuvent s'expliquer par les sensibilités au changement de la génération précédente. Les conflits très marqués entre les parents et les jeunes adultes en début d'union expliquent alors, par la confrontation, les références à des modèles familiaux très contrastés entre le mari et son épouse.

\section{Les différentes modalités du conflit : vers une interprétation de la place actuelle des femmes dans la famille paysanne amazonienne}

Pour comprendre le contexte des conflits observés, il faut au préalable connaître les outils de contrôle de la natalité dans les fronts pionniers. L'accès à ces outils est différent selon les âges et les rapports avec le mari. Disons que d'une manière générale, il existe deux pôles entre lesquels s'organisent les différentes techniques. D'un côté, il existe la méthode définitive qui consiste à réaliser une ligature des trompes des femmes (une stérilisation de l'homme est beaucoup plus rare). Cette méthode, légalement interdite aux femmes de moins de 25 ans et à celles qui n'ont qu'un seul enfant, peut se faire en ville, souvent à l'occasion d'un accouchement. Dans la mesure où c'est un outil définitif de limitation du nombre d'enfants dans la famille, son application fait l'objet de vives discussions dans les couples. La méthode qui serait son opposé est celle d'un contrôle «naturel » des naissances, c'est-à-dire sans acte médical, uniquement par une observation des cycles des courbes de température. Cette méthode est assez suivie dans le front pionnier où elle donne souvent satisfaction si le couple s'abstient de rapports pendant toute la période à risques : elle a l'énorme avantage d'être accessible à tous ceux qui ont une information, mais l'inconvénient de ne pas exclure des «accidents », volontaires ou pas. Entre ces deux opposés, les femmes peuvent utiliser les contraceptifs oraux, mais cela demande une certaine constance et l'accord du mari (gérant le budget, c'est lui qui décide de l'achat des contraceptifs), de même lorsque le couple décide d'utiliser des préservatifs.

Les techniques contraceptives ne sont d'ailleurs que des outils au service de volontés qui leur préexistent. Les historiens l'ont bien montré à propos de la France bourgeoise puis rurale du XVIII ${ }^{e}$ siècle. Ils rappellent que « la question du pourquoi (de la chute de la fécondité) est plus décisive que celle du comment. Le problème des moyens est subordonné à celui des fins, même si l'apprentissage des moyens introduit des décalages » [Bardet et Dupâquier, 1986 : 13]. Elles permettent de 
cristalliser, s'il y a conflit, des positions différentes. On observe trois figures du conflit : les deux premières portent sur la réalisation d'une ligature des trompes, soit que la femme estime avoir eu trop d'enfants, et veut profiter de cette technique pour ne plus en avoir, soit lorsqu'elle ne peut pas utiliser de méthodes contraceptives moins définitives car elle subit une pression constante de son mari pour avoir des enfants. La troisième figure, plus tragique, s'observe lorsqu'une femme décide d'utiliser des méthodes naturelles pour réduire le nombre d'enfants, mais qu'elle se voit contrainte par son mari à des rapports sexuels non protégés lorsqu'elle est fertile. Ces trois exemples emblématiques permettent de mesurer l'écart existant d'une part entre le nombre idéalisé d'enfants par l'homme et la femme, d'autre part, les représentations de l'enfant et de sa place dans la reproduction du modèle paysan, et enfin la place accordée à la parole féminine dans la famille paysanne.

\section{Trop d'enfants, trop de migrations... ou la nécessité d'une ligature des trompes}

Un premier type de conflit oppose des femmes qui ont déjà de nombreux enfants et qui souhaitent vouloir avancer la fin de leur vie fertile. Ces femmes sont issues de familles marquées par plusieurs migrations enclenchées lorsque les premiers enfants sont arrivés à l'âge adulte. Elles n'ont pendant longtemps pas eu accès aux méthodes contraceptives, ont été directement intéressées par ces techniques qui constituent, pour elles, une révolution, dans la mesure où il faut qu'elles affrontent des préjugés sociaux et l'opposition de leur mari. Les fronts pionniers étaient très marqués, et le sont encore largement, par les religions catholiques et évangélistes, pour lesquelles un enfant reste perçu comme un don de Dieu. Dans ce cadre, décider de ne plus avoir d'enfants peut apparaître comme une solution contraire à la Foi.

Dès lors, lorsqu'une femme déjà âgée, insérée dans la société paysanne locale, affirme vouloir pratiquer une ligature des trompes, elle a toutes les chances de s'opposer à sa famille. On l'observe dans le cas de Maria, une femme de 44 ans, mère de douze enfants :

M. : Mon mari n'a jamais voulu aller [chercher une terre] à cause de la vie qu'il menait, cette souffrance, parce qu'il buvait, il jouait. Tout ce qu'on mettait de côté, il l'utilisait pour lui. Alors, tous on travaillait dans les champs, et très souvent je laissais les enfants à la maison, pour pouvoir aller l'aider afin qu'il ne retire pas les enfants de l'école. Parce que je n'ai pas étudié, lui non plus d'ailleurs. Alors on ne sortait pas les enfants de l'école. J'allais l'aider, je préparais le riz, je m'occupais des champs. Alors il prenait et il vendait le riz, il jouait, il jouait aux dominos en pariant, il buvait de la cachaça, il allait à la fête, et il dépensait tout l'argent. Et tous les ans on avait un enfant, tous les ans un enfant. Il ne me laissait pas éviter.

Enquêtrice : Même pas cachée?

M. : Je ne pouvais pas acheter [de contraceptifs], alors je ne pouvais pas cacher. Tu me comprends ? Alors bon, ce petit-là, le dernier, il avait plus ou moins sept mois, et mon frère m'a demandé si je voulais faire une ligature des trompes. Alors j'ai dit que je voulais. Alors j'ai parlé avec ma mère pour qu'elle reste avec mes enfants, il y avait cinq petits. (...) Mais elle n'a pas voulu. Elle disait que non, parce qu'elle disait 
qu'elle avait beaucoup de travail et que si je faisais la ligature, j'allais mourir. Alors en colère j'ai dit : "Et bien puisque de toutes façons je dois mourir un jour, j'y vais quand même ». Et elle disait «Non ma fille, n'y va pas! » Alors j'ai été à sa maison, elle est restée avec mes enfants et j'y suis allée. (...) [Mais ça ne fonctionne pas, alors elle est obligée de se faire acheter des contraceptifs oraux par sa sœur en ville, et de les prendre en cachette. Elle raconte toutes les péripéties de la ligature]

E. : Vous pensez que les femmes ont le droit de choisir le nombre d'enfants ?

M. : Je pense bien que oui. Elles en ont le droit oui. Parce que souvent, la personne... la femme en veut un certain nombre, et le mari un autre nombre. Mais c'est la femme qui va souffrir...

On observe deux choses dans cet extrait d'entretien : tout d'abord, la vulnérabilité d'une femme face à son mari. Maria, mariée à un homme dont elle ne fait pas un portrait flatteur, ne semble pas en mesure d' «arrêter la fabrique d'enfants » [Blaffer Hrdy, 2002 : 367]. Bien qu'elle veuille espacer les naissances, elle reste dépendante de l'argent que lui procure son mari pour s'acheter des contraceptifs. À aucun moment n'apparaît la possibilité, pour elle, de contrôler sa fécondité.

Pour autant, Maria revendique le droit à disposer de son corps. Les relations mère / fille sont ici particulièrement intéressantes dans la mise à jour de ce moment de transition. L'attitude de la mère rend compte de l'intériorisation d'une référence à une forte fécondité comme idéalisation du modèle familial paysan et intériorisation d'une structure sociale. Pour autant le changement s'inscrit dans les failles de toute structure. L'attitude de Maria fait ainsi part de la distanciation qu'elle prend et nous interroge sur le moment de basculement entre le recevable et l'irrecevable : à quel moment les maternités répétées deviennent-elles inconcevables ? Elle s'interroge aussi sur le rapport à la douleur si naturellement associée à la mise au monde. Ces regards projetés se retrouvent dans les propos de femmes plus jeunes et en particulier de sa fille que nous rencontrerons plus loin.

\section{Une réduction forcée du nombre d'enfants... les regrets d'un mari}

Le second type de conflit observé se fait alors que le couple en est au début de sa vie procréative. Il s'agit du couple composé d'un homme de 44 ans marié à une jeune fille d'un peu moins de 25 ans. Les logiques biographiques de leurs entretiens montrent une forte différence entre un époux marqué par les logiques paysannes et une épouse qui veut s'en affranchir. Le conflit s'est matérialisé autour de la réduction du nombre d'enfants, ce dont parle en termes feutrés l'époux de ce couple :

Enquêteur : Vous vouliez avoir plus d'enfants?

José : Oui, je voulais, parce que j'aime beaucoup les enfants. (...) J'ai toujours voulu qu'elle ne fasse pas la ligature [des trompes] pour qu'il soit toujours possible que, quand on le décide, arrive une grossesse ; pas sans l'attendre, pas une grossesse inattendue, mais une grossesse que l'on a décidée, pour qu'il soit toujours possible d'avoir un autre fils. Mais ça n'a pas été possible.

E. : Comment faites-vous pour planifier?

J. : [Silence] Bon, on pense comme cela... ce fils, Junior, est né alors que nous étions mariés depuis un an... alors on a planifié la chose suivante : on veut un autre fils 
quand Junior aura trois ans, parce qu'il est déjà grand, ou quatre ans. De cette manière, il pourra surveiller l'autre pendant que sa mère fera les choses à la maison et il ira à l'école (...) Mais bon des fois on oublie le contraceptif pour éviter la grossesse, on faisait avec la petite table, toute cette histoire, parce que des fois le médicament elle ne se sentait pas très bien avec le médicament, alors on a utilisé la méthode suivante : durant la période fertile on devait observer un temps sans avoir de relations sexuelles, jusqu'à ce que passe la période fertile pour qu'il n'y ait pas de grossesse. Et toujours on a fait ainsi. Mais bon un jour, des fois, on... parce que je sens bien, ce que je t'ai déjà dit, je suis quelqu'un en bonne santé, grâce à Dieu, parce que je suis encore jeune, et je suis un homme pour une femme à n'importe quel moment (...) Alors rien ne me manque de ce côté-là, je suis quelqu'un c'est comme si j'avais 18 ans ou 14 ans. Je me sens ainsi, comme si j'avais toute l'énergie d'un jeune homme. Oui ? C'est pour cela, je pense que je suis un homme très fort de ce point de vue.

E. : Donc vous n'avez pas réussi à respecter la table, n'est-ce pas ?

J. : Ah oui, j'ai oublié de quoi on parlait. C'est pour cela, des fois on... bon elle a dit comme cela : «Non, on ne peut pas avoir des relations sexuelles, parce qu'on va avoir un autre enfant, et l'autre est encore petit, il ne peut pas s'occuper d'un autre ». Mais j'ai dit : «Mais non, tu as déjà passé la période, tu as perdu le compte, tu n'as pas compté les bons jours ». Elle a dit: «Je le sais mieux que toi »; mais je ne l'ai pas cru et... j'ai insisté, n'est-ce pas, et elle, pour ne pas me contrarier peutêtre, elle a accepté. Alors bon... un autre est venu, quand Junior a eu 2 ans José Neto est né.

Ce mari explique qu'il y a dans son couple une volonté d'espacement et de réduction des naissances, absente dans le cas précédent. La méthode utilisée est celle du suivi des cycles. José argumente le fait qu'il y a eu un accident qui explique la naissance de son second enfant plus tôt que prévu. Le récit de cette naissance révèle d'un côté l'insistance du mari, de l'autre, l'acceptation, sous pression pour l'épouse, de rapports non protégés.

Même si celle-ci (que nous connaissions moins bien que José) n'a pas évoqué cet incident, il est probable que cette pression soit à l'origine de la ligature des trompes qu'elle a effectuée en accouchant de son second enfant. Sous un prétexte médical (elle est atteinte de nausée pendant les trois premiers mois), nécessaire visà-vis de son mari et des médecins (elle a moins de 25 ans), elle a réalisé une ligature des trompes... que l'époux regrette.

Ce second cas montre d'un côté que la volonté de contrôle de leur corps par les femmes n'attend pas forcément un nombre élevé d'enfants et, d'un autre, que les hommes eux aussi sont amenés à composer avec les attentes de leur épouse. José est obligé de les accepter (au moins en façade), et sans doute de ruser pour les contourner ; avant finalement de voir cette dernière pratiquer une ligature des trompes. Ce conflit témoigne d'un changement très profond au sein des couples.

\section{Le viol conjugal, ou les rapports différents à la famille paysanne}

Le troisième cas de conflit se passe de récit direct. Il est le fait d'un couple qui s'est, à la différence des précédents, composé très rapidement: une semaine seulement 
sépare la première rencontre des fiançailles. Cette rapidité s'explique pourtant par des projets bien différents de l'un et de l'autre. La future épouse, alors âgée de 16 ans, vit dans une zone reculée du front pionnier et est soumise à l'autorité de son père qui ne la laisse pas avoir de petits amis. C'est pour en avoir eu un qu'elle est battue par son père et consignée chez elle à s'occuper de ses dix frères et sœurs pendant que sa mère est aux champs. C'est alors qu'elle voit arriver chez elle un jeune homme, à peine rentré d'une grande ville du Nordeste du pays qui lui demande un verre d'eau. Le jeune homme a en tête un projet précis : il est revenu de la ville pour s'installer comme agriculteur. L'accomplissement de ce projet suppose, selon lui, un mariage préalable. Le tableau de cette jeune fille s'occupant de sa nombreuse famille le ravit et apparaît conforme à son désir. Ce sont les attributs que ce jeune homme véhicule (urbanité et modernité) qu'elle soupèse quant à elle.

Évidemment, ce couple est particulièrement mal assorti. Les conflits commencent lorsque le nouvel époux veut partir vivre dans un lot reculé en forêt. Sa femme s'y oppose, et menace de divorcer s'ils quittent le lot où ils sont actuellement installés - le lot de son beau-père, situé à proximité de la ville. Le mari est obligé de céder, migrer sans épouse étant très difficile au point de vue de la logistique, et absurde au regard des logiques paysannes. Après ce premier échec, c'est sur l'autre base de la famille paysanne que va porter le conflit : le contrôle de la fécondité. L'épouse est enceinte très rapidement après le mariage, et dit aujourd'hui le regretter. Elle décide, afin de pouvoir continuer ses études, de ne pas avoir d'autres enfants avant plusieurs années. Son mari commence par accepter ; mais quatre ans après la naissance du premier enfant, alors que sa femme prépare les concours d'entrée à l'Université, le conflit éclate ouvertement.

La principale crise a lieu un soir : l'homme, qui sait que sa femme est ce soir-là fertile, tente d'avoir des rapports sexuels. Mais à la différence du cas précédent, ses avances sont refusées. Furieux, il quitte la maison. Son épouse se barricade et se couche. Au milieu de la nuit, l'homme, saoul, rentre, et, fou de rage de ne pouvoir ouvrir, enfonce la porte et se jette sur son épouse. La scène est interrompue par le surgissement de l'enfant du couple. Lorsque les deux protagonistes évoquent, en commun et séparément, cette scène, ils le font différemment. Chacun reconnaît que la maîtrise de la fécondité est au cœur du problème. Mais alors que l'époux se défend de toute action préméditée et parle - comme José - de pulsions profondes et de volonté d'avoir une sexualité qu'il qualifie de «normale », l'épouse parle de viol. Parler de viol dans le cadre d'un couple marié est un phénomène suffisamment rare pour être souligné.

À la différence des deux premiers témoignages, l'épouse impose à son conjoint la maîtrise de son corps et de sa fécondité, les deux étant, dans ces circonstances, intimement liés. Elle confirme en tout cas que les femmes qui ont un certain niveau d'éducation s'opposent à leurs maris, et peuvent leur imposer, dans certaines limites, des règles de conduite. Nous tenons là un élément de confirmation du rôle moteur des femmes dans la réduction de la natalité dans les fronts pionniers. Ces changements sont inévitablement porteurs de conflits car ils remettent «en jeu l'ordre social. Dans ce domaine intime, mais socialisé et contrôlé »[Segalen, 1980 : 139], le modèle social exige que les désirs des femmes soient soumis. 


\section{Interpréter les différentes formes du conflit : I'hypothèse d'un changement socio-spatial}

\section{Organisation de la place des uns et des unes et articulation des champs d'analyse}

Notre approche [Arnauld de Sartre, 2006 ; Granié et Guétat-Bernard, 2006], afin d'éclairer «ce qui ne va pas de soi », invite à penser que l'articulation des champs des individus et du quotidien oblige à une reconsidération des outils utilisés dans les autres champs. C'est en travaillant à l'échelle locale, intime, personnelle et quotidienne des relations au sein des familles restreintes de paysans que nous avons recueilli ces discours sur le corps, la sexualité, les conflits (y compris de représentations) à propos du nombre idéal d'enfants désirés par l'un et l'autre. Croiser ces champs, c'est tenter de toucher les interprétations individuelles selon que l'on est homme ou femme, et selon la place de chacun dans les organisations sociales et spatiales. Dès lors, si on admet que le corps et la sexualité peuvent devenir des sites de contrôle et de résistance [Aihwa, 1991], on comprend l'intérêt d'appréhender les liens entre les discours sur la fécondité et la reconduction d'un modèle paysan du front pionnier amazonien.

En considérant les figures du conflit autour de l'enjeu majeur de la réduction de la natalité, on peut dresser un tableau de ces changements et avancer des hypothèses quant aux modalités de leur diffusion, diffusion non seulement entre localités, entre familles dans une même localité mais aussi entre l'époux et son épouse au sein d'un même couple. L'accès plus facile aujourd'hui aux moyens contraceptifs révèle des conflits de représentations de la place de l'un et de l'autre au sein du couple et dans leurs relations au travail sur l'exploitation, mais aussi des évolutions sur la place accordée aux enfants dans le projet familial. Méfions-nous toutefois des contraintes auxquelles nous soumettons nos interlocuteurs dans la narration de leur vie. Des reconstructions a posteriori peuvent apparaître : l'objectif de réduction peut être ancien mais la volonté de limiter le nombre d'enfants a pu surgir tardivement, ce que la forme de l'entretien biographique ne permet pas de saisir. Car on peut supposer que si cette volonté était vraiment affirmée depuis longtemps, il est probable que les paysannes rencontrées auraient au moins réussi à espacer les naissances entre leurs enfants, et à en réduire le nombre.

Plus révélatrice est la tension qui sépare la position des mères et des filles. Elle paraît indiquer qu'un basculement s'opère entre les générations. Les normes sociales qui renvoyaient à un idéal de famille nombreuse sont fragilisées aujourd'hui. C'est particulièrement flagrant dans l'évolution des représentations du seuil de la famille nombreuse : d'une génération à l'autre le qualificatif de famille nombreuse passe de huit ou dix enfants à seulement quatre ou cinq. Lorsque cette réduction de la taille de la famille est présentée comme une volonté partagée du couple, les entretiens intergénérationnels dévoilent que cette volonté était déjà présente dans les intentions de la mère de l'époux, sans pour autant qu'il ait été possible pour elle de réaliser son désir. Ce serait donc un mouvement social progressif qui s'affirme aujourd'hui. La différence de comportement selon les 
familles (entre une réduction sans conflit et une fécondité maintenue élevée) révèle alors des variations à une norme du nombre maximal d'enfants en forte chute. L'accès plus aisé aujourd'hui à diverses méthodes contraceptives n'est venu que renforcer ces logiques de réduction de la descendance. Or cela est d'autant plus évident que cette question apparaît contemporaine d'un autre changement, celui de la place des enfants dans les familles paysannes.

\section{Statut de l'enfant dans la famille et travail féminin}

L'hypothèse d'un changement social permet d'éclairer les raisons avancées pour rendre compte de la réduction du nombre d'enfants. Milton en donne un aperçu :

E. : Et vous voulez plus d'enfants ?

M. : Écoute, je crois que l'on va s'arrêter là. Mon épouse n'a pas encore été opérée, moi non plus, mais quatre fils, je pense que c'est suffisant.

E. : C'est suffisant?

M. : Oui, je pense que oui. La crise dans laquelle nous nous trouvons aujourd'hui, on... il y a des gens qui ont beaucoup d'enfants, mais ils n'ont pas les moyens de leur donner des études, de s'en occuper. Moi, je donne des études à mes enfants... et je vais voir si je suis... je vais leur donner des études tant que je le pourrais, mais on ne peut pas tout faire pour eux parce qu'on n'a pas les moyens, n'est-ce pas ? Si c'est de la volonté de Dieu aussi...

Ce discours, comme une autre expression entendue : «moins d'enfants pour mieux s'en occuper », justifie concrètement la réduction du nombre d'enfants et un effort porté sur les études.

Les travaux des historiens sur l'idée de l'enfance en Europe peuvent aider à interpréter cet argument. Ceux de Philippe Ariès [1979] ont bien sûr été précurseurs par la mise en correspondance de la transition démographique (en France dans la seconde moitié du XVIII ${ }^{\mathrm{e}}$ siècle) avec la montée de l'individu accompagnant une « émergence croissante de la sphère du privé » [Julia, 1998 : 47] et d'un « besoin d'intimité et d'identité » désormais associé à une nouvelle idée du bonheur [Mauzi, 1979]. Ces deux phénomènes auraient justifié une importance croissante accordée à l'éducation des enfants [Becchi et Julia, 1998 : 25]. Ceux-ci n'étant plus considérés comme une partie du patrimoine de la famille, destinés à assurer sa reproduction mais comme des sujets à part entière, l'éducation formelle devait les conduire à être maîtres de leur destin.

L'histoire européenne nous apprend que la diffusion de valeurs culturelles nouvelles marquant un changement des normes familiales autour de la «famille moderne » a connu dans la société rurale une diffusion rapide (au moment de la Révolution française et des nouvelles règles du droit d'héritage) confortant l'idée que la volonté de réduction du nombre d'enfants a aussi été liée paradoxalement à celle «délibérée de confort personnel et de maintien des patrimoines » [Bardet et Dupâquier, 1986 : 16]. Le souci de préservation ou d'ascension sociale a encouragé la famille paysanne à restreindre sa descendance finale tout en fragilisant, par l'accès au monde urbain et à ses références éducatives, les chances de reproduction 
du modèle paysan. « Dès qu'elles eurent la possibilité d'élargir l'horizon de leurs prévisions, les familles tentèrent de se constituer un certain capital » [Challier, 1986 : 76] expliquant une manière nouvelle de se projeter dans un horizon temporel et par là même territorial.

Sur le front pionnier, la réduction du nombre d'enfants peut être associée comme dans le passé européen à un nouveau regard porté sur leur place dans la famille : tout en s'accompagnant d'une plus grande attention portée à leur instruction, il prépare à une sortie de l'agriculture paysanne [Arnauld de Sartre, 2005] et à ses modes de reproduction par la migration. L'augmentation moyenne du niveau scolaire est patente : alors que rares sont les agriculteurs âgés de plus de 50 ans qui savent lire et écrire, tous les jeunes ont un niveau au moins de fin d'école primaire et $33 \%$ d'entre eux ont étudié au moins deux années au collège, souvent en ville.

Reprenons les explications des historiens lorsqu'ils nous apprennent que « l'ampleur des variations dans la taille des groupes domestiques est réduite » dans le temps long de l'histoire européenne. Jack Goody propose plutôt de reporter à la Révolution industrielle les changements majeurs dans la structure des familles lorsque la «dépendance jusque-là étroite entre vie familiale et économie domestique se relâche » [2001 : 241]. Plus précisément, les bouleversements économiques du XIX ${ }^{\mathrm{e}}$ siècle sont à la fois cause et conséquence de la distinction désormais marquée entre le domestique et la consommation associés au monde des femmes, opposés à la production et au travail liés à celui des hommes [Perrot, 1998]. Une figure idéalisée du mari et père assurant seul le lien à l'économie marchande se façonne alors. L'emploi des hommes à l'extérieur de l'espace domestique acquiert le qualificatif de productif. En contrepoint, sont érigées en seules «travailleuses » les salariées, alors que boutiquières et paysannes sont reléguées dans l'ombre de l'auxiliariat conjugal [Perrot, 1998: 191] déconsidérant le travail féminin en l'associant à la seule figure de la «femme qui aide » particulièrement dans les campagnes [Verdier, 1979] et tout en érigeant en vertu le rôle de la ménagère. Ce qualificatif de celle qui aide est toujours présent dans la représentation qu'hommes et femmes donnent du travail féminin sur l'exploitation, et ce aussi bien dans les campagnes françaises que dans les fronts pionniers où nous travaillons [Garcia, 1983].

Mais la réalité des faits dans l'histoire européenne s'est avérée bien différente : l'investissement des femmes dans les activités qualifiées désormais de productives s'est maintenu par nécessité pour les classes populaires et avec force dans les travaux de la terre. Car quoiqu'en ait conservé la mémoire collective, et malgré les « silences de l'histoire » [Perrot, 1998], les femmes ont continué à travailler, y compris à l'extérieur de la « sphère » privée, désormais pourtant accolée par nature à l'idéal féminin [Laqueur, 1992]. Nous touchons là au cœur de la compréhension du phénomène : c'est dans les relations homme / femme au sein des couples, en lien avec les changements dans les représentations de soi, et de soi dans un rapport social, que se lisent les bouleversements. Les tiraillements dans les difficultés de gestion tout à la fois du dedans et du dehors expliquent un désir et la mise en pratique d'une conduite malthusienne par les femmes de ce temps passé. Se mêlent 
alors dans la compréhension de la baisse tendancielle du nombre d'enfants par la femme dans l'histoire européenne tout autant un nouveau regard porté sur l'enfance, qu'un désir qui s'affirme d'accomplissement personnel, mais aussi et peut-être surtout une tension forte entre des responsabilités et des temps de travail que l'évolution socio-économique et politique imposent aux femmes. Toute l'ambivalence repose d'ailleurs sur l'impossibilité de « séparer l'histoire du travail féminin de l'histoire de la famille, des rapports de sexe et des rôles sociaux » [Perrot, 1998 : 194]. Les extraits d'entretien ici proposés montrent bien que toutes ces projections sont mêlées dans la compréhension que les paysannes se donnent du monde et de la place qu'elles souhaitent y tenir. Qu'elles souhaitent s'intégrer dans l'univers paysan, qu'elles tentent d'articuler travail sur l'exploitation et hors de celle-ci par un travail d'institutrice par exemple, ou encore qu'elles mettent en œuvre des stratégies pour échapper aux contraintes de vie sur l'exploitation, les femmes qui nous ont fait part de conflits touchant à la programmation du nombre idéal d'enfants l'ont associé à une tentative de reconnaissance de leur compétence et de leur temps de travail.

La situation que dévoile Zilda, fille de Maria (précédemment présentée) est particulièrement révélatrice des changements qui s'opèrent. Elle est mariée depuis sept ans à un jeune homme de son âge lui-même issu d'une grande famille de onze enfants (Zilda a également, rappelons-le, onze frères et sœurs), plus modeste économiquement que celle de Zilda ; il est simple vacher lorsqu'ils se rencontrent. Elle apparaît comme une jeune femme décidée qui a choisi de s'installer en agriculture après un passage de quatre ans en ville. Cette installation n'est pas le simple résultat de son engagement marital ; d'ailleurs le passage par la ville ayant été vécu difficilement, c'est elle qui décide de revenir à la terre en s'installant près de ses parents. Elle achète ainsi 25 hectares en son nom, et avec son mari en gère 25 autres qui appartiennent au père de Zilda. Les crédits pour la construction de la petite maison dans laquelle ils vivent non loin du lot des parents sont également en son nom. Au début de sa vie active, Zilda fait ainsi part d'une projection différente de celle de sa mère. En début d'entretien, contrairement à l'histoire douloureuse qu'a subie sa mère en raison de l'alcoolisme de son père, elle exprime fermement la nécessité d'une entente entre les époux :

Z. : C'est cela même, il faut trouver un accord, parce qu'on doit tenter de s'articuler, et, si ça marche, continuer ensemble. Il faut que ça aille bien sinon on laisse tomber.

E. : Combien d'enfants voulez-vous ?

Z. : Pas un de plus, juste ces quatre-là, puisque Dieu a voulu emporter les deux autres. Mais maintenant, simplement ces quatre.

E. : Vous n'allez pas insister plus ?

Z. : Non, simplement si par hasard cela devait arriver, mais on n'en veut pas plus.

E. : Mais vous faites des contrôles, comment procédez-vous ?

Z. : Non.

E. : Vous ne prenez pas de contraceptifs, des choses de ce genre?

Z. : Ah non, cela doit dépendre de la volonté de Dieu. 
E. : Mais vous savez que vous pouvez contrôler, qu'il existe des possibilités de faire cela, vous avez toutes les informations à ce sujet, n'est-ce pas ?

Z. : Oui, on sait.

E. : Et Raimundo, il en pense quoi ?

Z. : Il est d'accord qu'on a déjà assez d'enfants.

E. : Donc vous ne voulez pas en avoir douze comme votre mère?

Z. : Ah non, on ne veut pas, non.

E. : Pourquoi ?

Z. : On travaille, et c'est très difficile. De nos jours pour que des parents puissent élever douze enfants c'est difficile, très difficile, et moi je sais que mon père a eu beaucoup de mal à nous élever, ça lui a demandé beaucoup de travail, de difficultés. (...) Nous on n'a pas fini nos études, moi je n'ai pas terminé parce qu'on est venus ici, et on a passé beaucoup de temps bloqué ici, et bon c'était déjà l'époque pour moi d'avoir des petit-amis, et bon, je me suis mariée et je n'ai pas terminé, parce qu'une fois que j'étais mariée mon mari ne m'a pas laissé terminer mes études.

E. : Comment cela votre mari ne vous a pas laissé ?

Z. : Parce qu'il ne voulait pas me laisser, c'est difficile quand on a des enfants parce qu'il devait rester à la maison pour les surveiller parce que je devais étudier le soir, et il ne voulait pas rester à la maison avec les enfants pour que je puisse étudier et alors c'est difficile quand on est femme au foyer, sauf si on a vraiment très envie, là oui c'est possible, mais c'est difficile. Mais j'ai très envie de terminer mes études et de déménager, en ce moment même je suis en train de vendre un terrain, mais bon ce sera pour quand les enfants seront grands, qu'ils puissent nous aider un peu.

Dans cet extrait d'entretien et dans son attitude corporelle lors de la rencontre, Zilda fait preuve de beaucoup d'assurance et de maturité dans sa ténacité à mener à bien son projet de vie sur la terre. Elle apparaît comme étant entièrement partie prenante des choix pris par le couple; elle indique d'ailleurs que c'est la seule condition de réussite conjointe du projet familial et du projet d'exploitation. Elle révèle ainsi un engagement qui est décelable déjà chez sa mère. On voit alors comment s'articulent son désir de limitation du nombre d'enfants et son engagement professionnel sur l'exploitation et dans la localité (on lui propose de devenir la secrétaire de l'association aux prochaines élections). Elle parle aussi clairement d'un lien entre un grand nombre d'enfants et les difficultés de les tenir aux études. Elle regrette elle-même d'avoir dû arrêter trop tôt et de s'être mariée rapidement, d'autant que même si une grande sérénité transparaît dans ses propos et son attitude, elle souhaiterait que son mari la laisse suivre les cours du soir pour adultes afin de parfaire son éducation. La scolarisation est devenue une valeur essentielle pour la paysannerie et compte beaucoup pour le contrôle de la natalité. C'est autant la place de l'enfant dans la famille et son rapport nouveau à la société urbaine au travers notamment de l'éducation, que l'engagement que défendent les paysannes rencontrées qui apparaissent comme des éléments explicatifs de désir ou d'une réelle capacité de mise en œuvre d'une réduction de la natalité. 


\section{Conclusion : I'hypothèse d'un changement de contexte}

La valorisation de la fécondité - signe de la puissance sexuelle masculine, du pouvoir génésique des femmes [Lecarme, 1999], de la bénédiction divine, de la reconduction d'un modèle paysan - est à ce point intériorisée qu'elle apparaît indissociable du destin des femmes. Leur « domestication sociale » n'est jamais assurée et leur rébellion toujours menaçante légitimerait jusqu'aux violences physique, symbolique et imaginaire exercées sur elles. Mais le consentement ou la résignation à l'inéluctable ne l'est plus toujours aujourd'hui. Même si le pouvoir symbolique des femmes, positif et négatif, inséparable de leur pouvoir génésique, repose sur leur adhésion à la croyance dans le «travail de la mère », si celui-ci est alors présenté comme un impératif moral catégorique [Lecarme, 1999] justifiant les éléments de l'éthos féminin - le service, le sacrifice - lorsque l'on prend le temps de les interroger, les paysannes ne gardent pas le silence et font part de leurs projets.

Les ruralistes qui s'engageaient dans l'étude du changement social dès les années 1960 ont montré l'intérêt de l'articuler avec les structures mêmes des sociétés rurales qu'il touche [Gervais \& al., 1977]. En ce sens, les évolutions de contexte dans lequel s'inscrivent les fronts pionniers amazoniens doivent éclairer les logiques propres aux familles paysannes rencontrées, et au sein de ces logiques les interprétations personnelles que font de ces évolutions les paysans et les paysannes. Car les explications à cette baisse brutale et rapide du nombre d'enfants, tellement brutale et rapide que tous les couples ne s'y résignent pas, doivent être affinées. C'est cette compréhension des interactions entre les logiques individuelles, celle des groupes domestiques et le contexte spatial, social, économique et historique qui doit alors être poursuivi. C'est en suivant cette voie qu'il nous sera possible de comprendre les relations mutuelles qu'entretiennent les changements démographiques qui affectent les individus et les groupes domestiques dans lesquels ils résident [Ségalen, 1996 : 39] et les changements des rapports spatiaux. Notre explication pourra alors s'inscrire dans une proposition qui tentera d'éclairer les liens entre individus hommes et individus femmes / groupes familiaux d'appartenance / contexte global d'action. Dans cette perspective, la compréhension des liens entre l'organisation des groupes domestiques et les tensions révélées sur les choix reproductifs est à approfondir.

C'est aussi la diffusion du modèle urbain qui est ici questionnée et surtout l'interprétation qu'en font les familles paysannes, en particulier dans ces caractéristiques de baisse de la natalité, d'une augmentation du niveau moyen de scolarité, et plus encore d'une dynamique d'individuation centrée sur le désir d'accomplissement personnel. L'hypothèse d'un changement des dynamiques de colonisation de la forêt amazonienne s'en trouve renforcée. Jusqu'à présent, l'intégration d'un front pionnier au reste du Brésil ne se traduisait pas par une baisse de la natalité, celle-ci restant élevée sur l'ensemble du territoire brésilien. Mais la première ayant commencé à décliner fortement il y a trente ans, le phénomène est en cours de diffusion dans le front pionnier - avec le retard caractéristique de ces régions. Cela pourrait avoir des conséquences non seulement sur le mode de vie paysan, mais aussi sur le rapport à la durabilité de cette agriculture paysanne. 


\section{BibliographIE}

Ainwa O. [1991] «The Gender and Labor Politics of Postmodernity », Annual Review of Anthropology, $\mathrm{n}^{\circ}$ 20, p. 279-309.

ARIES P. [1979] Histoire des populations françaises et de leurs attitudes devant la vie depuis le XVIII siècle, Paris, Seuil : 414 p.

ARNAULD DE SARTRE X. [2005] «Les jeunes filles des fronts pionniers amazoniens: «passeuses » du changement et négociatrices d'une nouvelle ruralité ? », in G. Capron, G. Cortes et H. Guétat (Dir.), Liens et Lieux de la Mobilité. Ces autres territoires, Paris, Belin, p. 29-43.

ARNAULD DE SARTRE X. [2006] Fronts pionniers d'Amazonie. Les logiques paysannes au Brésil, Paris, CNRS Éditions, $227 \mathrm{p}$.

BARDET J.-P. et DuPAQUIER J. [1986] «Contraception: les Français les premiers, mais pourquoi ? », Communications, $\mathrm{n}^{\circ} 44$, p. 3-32.

BECCHI E. et Julia D. [1998] «Histoire de l'enfance, histoire sans paroles ? », in Histoire de l'enfance en Occident, tome I. De l'antiquité au XVII' siècle, Paris, Seuil, p. 7-41.

BlafFer HRdY S. [1999] Les instincts maternels, Paris, Payot, 622 p.

Brumer A., Dugue G., Lourenço F.A. et BAudel Wanderley M.N. [1991] «L'agriculture familiale au Brésil », in $\mathrm{H}$. Lamarche (dir.), L'agriculture familiale : une réalité polymorphe, Paris, L'Harmattan, p. 159-210.

CHALliER M.-C. [1986] « Du malthusianisme ascétique à l'économie familiale (1680-1914)», Communications, $\mathrm{n}^{\circ} 44$, p. 71-83.

GARCIA Jr. A. R. [1983] Terra de trabalho., Rio de Janeiro, Paz \& Terra, 236 p.

Gervais M., Jollivet M. et TAVERnier Y. [1977] «La fin de la France paysanne », in G. Duby et A. Wallon (Dir.), Histoire de la France rurale, Tome IV : La fin de la France Paysanne, Paris, Seuil, 755 p.

Goody J. [2001] La famille en Europe, Paris, Seuil, 283 p.

GRANiE A.M. et GuETAT-BERNARD H. (Dir.) [2006] Empreintes et inventivités des femmes dans le développement rural, Paris/Toulouse, IRD/PUM, coll. Ruralités Nord/sud, $350 \mathrm{p}$.

HaLl A.L. [1989] Developing the Amazons : deforestation and social conflict in Brazil's Carajás programme. Manchester : Manchester University Press, $220 \mathrm{p}$.

Hamelin P. [1992] «Mutation au Brésil. Vue d'Amazonie », Cahiers des Sciences Humaines, vol. $28, \mathrm{n}^{\circ} 4$, p. $727-748$.

Heritier F. [1996] Masculin / Féminin. La pensée de la différence, Paris, Odile Jacob, 332 p.

JuLIA D. [1998] «L'enfance entre absolutisme et lumières (1650-1800), in Histoire de l'enfance en Occident, tome II. Du XVIII à nos jours, Paris, Seuil, p. 8-119.

LAQUEUR T. [1992] La fabrique du sexe. Essai sur le corps et le genre en Occident, Paris, Gallimard, $355 \mathrm{p}$.

LECARME M. [1999] "La "fatigue" des femmes, le "travail de la mère" en milieu populaire dakarois », in D. Jonckers, R. Carré, M.-C. Dupré (Dir.), Femmes plurielles. Les représentations des femmes, discours, normes et conduites, Paris, Ed. de la MSH, 300 p.

LENA P. [1999] « La forêt amazonienne : un enjeu politique et social contemporain », Autrepart, $\mathrm{n}^{\circ}$ 9, p. 97-120.

LENA P. et MACIEL da SilveIRA I. [1993] Uruará : o futuro das crianças numa área de colonização, Belém, UFPa - UNAMAZ, 92 p.

MAUZI R. [1979] L'idée du bonheur dans la littérature et la pensée françaises au XVIII siècle, Paris, A. Colin, 383 p.

MendRAs H. [2000] «L'invention de la paysannerie. Un moment de l'histoire de la sociologie française d'après-guerre », Revue française de sociologie, vol. 41, n 3, p. 539-552. 
Perrot M. [1998] Les femmes ou les silences de l'histoire, Paris, Flammarion, 491 p.

Sebille P., Arnauld de Sartre X., Droulers M., Cosio-Zavala M.E. [2005] Enquêtes biographiques en Amazonie brésilienne, Rapport de recherche de l'ACI Société et culture dans le développement durable, Ministère de la recherche, 88 p.

SEgalen M. [1980] Mari et femme dans la société paysanne, Paris, Flammarion, 211 p.

VERDIER Y. [1979], Façons de dire, façons de faire. La laveuse, la couturière, la cuisinière, Paris, Gallimard, Bibliothèque des sciences humaines, $347 \mathrm{p}$. 